\title{
Pregnancy and death: An examination of pregnancy- related deaths among adolescents in South Africa
}

\author{
$\mathrm{N}$ de Wet, $\mathrm{PhD}$ \\ Demography and Population Studies Programme, University of the Witwatersrand, Johannesburg, South Africa
}

Corresponding author: Nicole de Wet (nicole.dewet@wits.ac.za)

\begin{abstract}
Background. South Africa (SA)'s high adolescent fertility has been extensively studied. A pregnancy outcome that has not received sufficient attention in research is the causes of death among pregnant adolescents.

Objectives. To examine levels and causes of adolescent maternal mortality in SA.

Methods. A secondary data analysis of Death Notification Forms from 2006 to 2012 was carried out. SA General Household Surveys from 2006 to 2012 were used to ascertain the number of female adolescents in the population. Frequency distributions and life table techniques were employed.

Results. An estimated 1164 deaths have been recorded among pregnant adolescents between 2006 and 2012. Adolescent maternal and pregnancy-related mortality is lower than adult maternal and pregnancy-related mortality. The main causes of death among adolescents were hypertension (55.6\% of all direct causes), abortion ( $17.6 \%$ of all indirect causes) and injuries ( $48.9 \%$ of all indirect causes). The probability of adolescents dying while pregnant without these causes is practically non-existent (range $0.002-0.150 \%$ ).

Conclusion. Policies and programmes should prioritise these pregnancy-related causes of death in order to further reduce such deaths among adolescents in SA.
\end{abstract}

S Afr J Child Health 2016;10(3):151-155. DOI:10.7196/SAJCH.2016.v10i3.978

Maternal mortality in sub-Saharan Africa (SSA) is higher than in any other region in the world. In countries such as Sierra Leone it is as high as 1100 per 100000 live births. ${ }^{[1]}$ The National Committee for Confidential Enquiry into Maternal Deaths in South Africa (SA) reports the current maternal mortality rate at 147.7 deaths per 100000 live births. ${ }^{[2]}$ Avoidable factors, including patient-related non-compliance $(49 \%)$ and healthcare and health worker factors (35\%), are contributing to maternal mortality. ${ }^{[3]}$ The National Department of Health (NDoH) has made attempts to reduce this, such as the implementation of a programme to reduce child and maternal mortality through strengthening primary healthcare in the country. While progress has been made - maternal mortality has decreased from 299 deaths per 100000 live births in 2007 to 147.7 in 2013, SA still failed to meets its Millennium Development Goal of 38 deaths per 100000 live births. ${ }^{[4]}$ In SA, alarmingly high rates of adolescent fertility are a topical issue. ${ }^{[5]}$ Despite teenage pregnancy rates decreasing from 116 per 1000 women (aged 15 - 19 years) in the 1980s, an astonishing 30\% of adolescent females still report 'ever being pregnant. ${ }^{[6,7]}$ While research has identified the levels and determinants of adolescent fertility, ${ }^{[8]}$ the issue of adolescent maternal and pregnancy-related deaths has yet to be addressed. Pregnant adolescents face stigmatisation and are unable to afford healthcare, ${ }^{[9]}$ which reduces healthcare accessibility and increases the risk of pregnancy complications and mortality. The extent of this is unknown.

The objective of this article is to determine the level and assess the direct and indirect causes of adolescent maternal mortality in SA. The health and survival of adolescent females is an important development goal of the national government. Adolescents who transition into healthy and productive adults are a key social and labour resource for any country. For this reason, research addressing the levels and causes of adolescent mortality will contribute to reducing deaths and ensure the health and survival of adolescents in the country.

\section{Methods}

Maternal death is the death of a woman while pregnant or within 42 days of termination of pregnancy, irrespective of the duration and site of the pregnancy, from any cause related to or aggravated by the pregnancy or its management but not from accidental or incidental causes. ${ }^{[10]}$ Pregnancy-related death is defined as the death of a woman while pregnant or within 42 days of termination of pregnancy, irrespective of the cause of death. ${ }^{[10]}$ This study examines both maternal deaths (direct maternal causes of death) and pregnancyrelated deaths (all deaths including those from accidental or incidental causes referred to as indirect causes of death) but not those which occurred in the post-natal period ( 42 days within termination of the pregnancy). The study seeks to answer the question: What are the levels and causes of maternal and pregnancy-related deaths among adolescents in SA?

The study was set in SA, where adolescent pregnancies are high and generally adolescent females have higher odds of mortality than adolescent males. ${ }^{[7,11]}$ The study was an analysis of secondary data available in the public domain. All deaths recorded on Death Notification Forms (DNFs) from 2006 to 2012 were analysed. Adolescent females who were pregnant at the time of their death $(n=1164)$ were analysed. All completed forms where the deceased was female and pregnancy status was confirmed were included in the study. The percentage of missing cases for pregnancy status ranged from $1.41 \%$ to $32.44 \%$ over the period. This percentage was checked against direct maternal causes of death (ICD-10 codes O00 - O99) - 8.73\% ( $n=966$ of the total 8433 maternal deaths of females of all ages) were found incomplete. These forms were not included in the analysis. The data were anonymised by Statistics SA before becoming available for public download. The data were not collected by the author and there was no need for institutional review board approval.

Analysis of direct 'Maternal Conditions' (ICD-10 codes O00 O99) and indirect causes of death were conducted to quantify the extent to which disease and injuries are contributing to adolescent maternal and pregnancy-related deaths in the country. Direct causes of death include conditions related to the pregnancy, such as maternal haemorrhage, maternal sepsis, gestational hypertension, obstructed labour and abortion. Indirect causes of death include communicable diseases that are not related to maternal or neonatal health, noncommunicable diseases, injuries and causes of death that were coded 
as ill-defined. The latter coding is checked on forms when a definitive cause of death could not be determined. Conventional multiple decrement life table techniques were done to estimate the probability of dying from direct and indirect causes of death among pregnant females in the country. In doing so, the study aimed to estimate the probability that a pregnant female will die from causes of death both related and unrelated to the pregnancy. Population age distribution was taken from the SA General Household Surveys from 2006 to 2012.

\section{Results}

The maternal mortality ratio in adolescents $(<19$ years) and adults ( $>20$ years) was approximated from an analysis of the pregnant female's age and the 1047886 live births to adolescents during the period and application of the same proportion to all live births ( $n=7320$ 968) during the study period. ${ }^{[12]}$ The adolescent maternal mortality ratio was 1.11 deaths per 100000 live births, while the adult maternal mortality ratio was 2.03 deaths per 100000 live births. Throughout the period, adolescent maternal mortality rates were consistently lower than those of adult females (Fig. 1). While rates of adolescent and adult maternal mortality appear to have peaked in 2009 , these have been consistently declining ever since.

Proportional mortality ratios (PMR) were estimated using the total number of female adolescent deaths $(n=13$ 930) and all female deaths $(n=41047)$ from 2006 to 2012 . Hypertension $(55.60 \%)$ was the leading medical cause of death among adolescent females in SA (Table 1). Hypertension contributed 24.09\% PMR to the overall mortality of pregnant adolescent females. In addition, abortion accounted for $17.60 \%$ of deaths in pregnant adolescent females over the period. Further, the PMR for deaths from abortion was $7.63 \%$. Overall, direct causes of death contributed $43.33 \%$ PMR of all pregnant adolescent deaths.

All other communicable deaths contributed $25.08 \%$ of deaths with a PMR of $14.21 \%$. Injuries contributed almost $50 \%$ of all pregnant adolescent deaths. More specifically, homicide and violence contributed $6.25 \%$ of all deaths, and all other forms of nondisease causes of death (other) have a PMR of $25.30 \%$.

Hypertension is the leading direct cause of maternal mortality for adolescent and adult females, at 13.61 deaths per 100000 live births overall (Fig. 2. (A)). In addition, hypertension causes more deaths in adolescents (13.26 deaths per 100000 live births) than adult females (11.38 deaths per 100000 live births) in the country. Alternatively, there are more adult deaths owing to abortion than adolescent deaths,

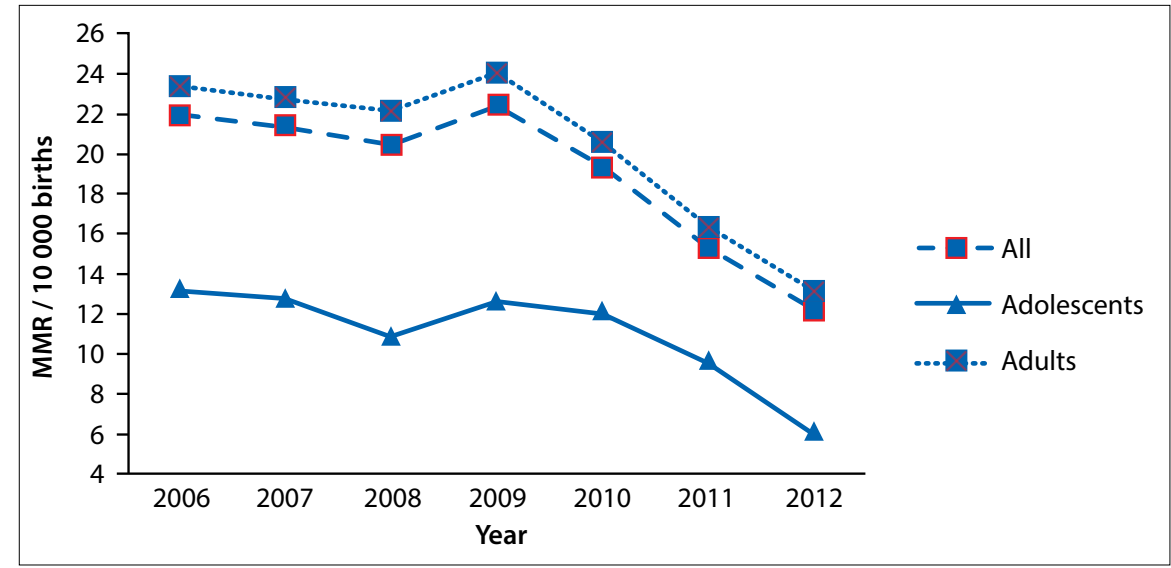

Fig. 1. Trends in maternal mortality ratio (MMR) by age of the mother and year of death, $2006-2012$.

Table 1. Distribution of adolescent maternal mortality by cause of death, 2006 - 2012

\begin{tabular}{llll}
\hline Cause of death & Deaths, $n$ (\%) & PMR, $\%$ & $p$-value \\
\hline Direct causes & & & \\
Maternal haemorrhage & $23(9.2)$ & 3.99 & 0.000 \\
Maternal sepsis & $43(17.2)$ & 7.45 & \\
Gestational hypertension & $139(55.6)$ & 24.09 & \\
Obstructed labour & $1(0.4)$ & 0.17 & \\
Abortion & $44(17.6)$ & 7.63 & \\
Sub-total & $250(100)$ & 43.33 & \\
Indirect causes & & & \\
Communicable (excl. maternal and neonatal) & $82(25.1)$ & 14.2 & 0.000 \\
Non-communicable & $60(18.4)$ & 10.4 & \\
Injuries & $160(48.9)$ & 27.7 & \\
Suicide and self-inflicted & $4(2.5)$ & 0.7 & \\
Homicide and violence & $10(6.3)$ & 1.7 & \\
Other (transport, accidents, etc.) & $146(91.3)$ & 25.3 & \\
Ill-defined & $25(7.7)$ & 4.3 & \\
Sub-total & $327(100)$ & 56.7 & \\
Total & 577 & 100 & \\
& & &
\end{tabular}

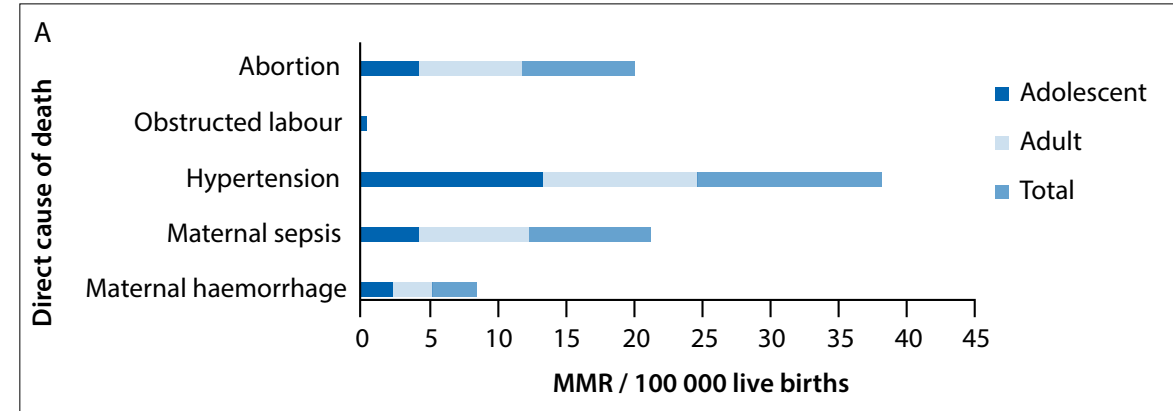

Fig. 2. (A) MMRs by direct medical cause of death, SA, 2006 - 2012.

with maternal mortality ratios of 7.56 and 4.20 , respectively.

In terms of indirect causes of death, communicable causes of death, excluding obstetric (maternal and neonatal) causes, are the highest contributor to overall pregnancy- related mortality ratios (37.56 deaths per 100000 live births). However, injuries are the highest indirect cause of death among pregnant adolescent females, at 15.27 deaths per 100000 live births, compared with the 10.11 adult deaths per 100000 live births 
from the same cause (Fig. 2.(B)). Finally, non-communicable diseases are higher among adult females (15.94 deaths per 100000 live births) than adolescent females (5.73 deaths per 100000 live births) in the country.

The probability of dying $\left({ }_{n} q_{\mathrm{x}}\right)$ and probability of dying if direct and indirect causes were eliminated $\left({ }_{\mathrm{n}} q_{\mathrm{x}}^{-\mathrm{i}}\right)$ from the pregnant female population in SA are seen in Table 2.

For young adolescents (10 - 14 years old) the probability of dying in general $\left.{ }_{n} q_{\mathrm{x}}\right)$ during pregnancy is practically non-existent $(0.0001$ or $0.01 \%)$ and the probability drops even further in the absence of direct $(0.002 \%)$ and indirect $(0.007 \%)$ causes of death. The overall probability of dying is higher for older pregnant adolescents at $0.15 \%$; however, when all direct and indirect causes are eliminated, the probability of dying for this age group is practically nonexistent. While the probabilities are higher for older adolescents who are pregnant, at $0.15 \%$ overall probability of dying, the probabilities decrease with the elimination of direct and indirect causes. Adult females have consistently higher probabilities of dying during pregnancy, and again in the absence of direct and indirect causes of death, the probability of dying is very low.

\section{Discussion}

The objective of this article was to learn the levels and causes of adolescent maternal mortality in SA. Adolescent maternal mortality rates are lower than adult rates in SA. This is similar to results of a study in Mozambique, which found adolescents to contribute to $22 \%$ of all maternal mortality. ${ }^{[13]}$ In other parts of Africa, pregnancy is a leading cause of adolescent female mortality, with rates much higher than in SA and Mozambique. ${ }^{[14]}$ In countries such as Chad, Guinea, Mali and Niger, high prevalence of child marriage and early pregnancy (with about half of adolescents giving birth before the age of 18 years old) is perpetuating adolescent maternal mortality ${ }^{[15]}$ In SA and Mozambique, child marriage is not nearly as prevalent as elsewhere, and this could explain the reduced contribution of adolescents to overall maternal mortality in these countries. Of notable importance in SA, however, is the rate of teenage pregnancies, which is high at approximately $11 \%{ }^{[16]}$ The mean age of pregnancy among adolescent females in SA is 18 years. ${ }^{[17]}$ Research has found that older adolescents are at a lower risk of maternal mortality and morbidity; this could explain why adolescent maternal mortality is lower in SA ${ }^{[18]}$ Further, in a study examining the selfreported sexual behaviours of youth, it was found that termination of pregnancy rates had increased from $5.1 \%$ in 2002 to $6.0 \%$ in

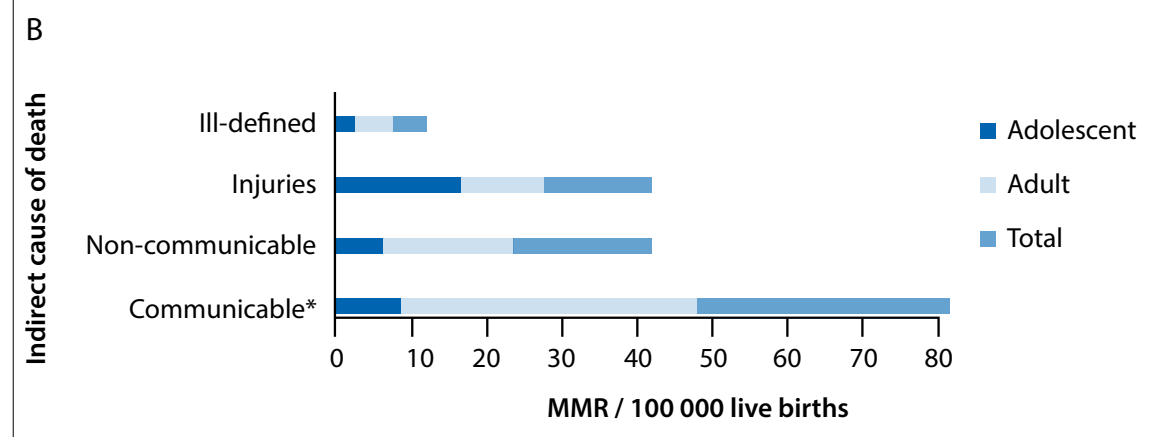

Fig. 2. (B) MMRs by indirect medical cuase of death, SA, 2006 - 2012. ( ${ }^{*}$ excludes maternal causes.)

Table 2. Probability of dying with and without main direct and indirect cause of death among pregnant women, $2006-2012$

\begin{tabular}{lllll}
\hline & & & \multicolumn{2}{c}{${ }^{n} q^{-\mathrm{i}}$} \\
\cline { 4 - 5 } Adolescents & Age group & ${ }_{\mathrm{n}} \boldsymbol{q}_{\mathrm{x}}$ & Direct & Indirect \\
\hline \multirow{3}{*}{ Adults } & $10-14$ & 0.0001 & 0.00002 & 0.00007 \\
& $15-19$ & 0.0015 & 0.00032 & 0.00149 \\
& $20-24$ & 0.0039 & 0.00078 & 0.00394 \\
& $25-29$ & 0.0058 & 0.00125 & 0.00581 \\
& $30-34$ & 0.0061 & 0.00143 & 0.00611 \\
& $35-39$ & 0.0043 & 0.00107 & 0.00429 \\
& $40-44$ & 0.0019 & 0.00063 & 0.00188 \\
& $45-49$ & 0.0009 & 0.0005 & 0.00088 \\
$50-54$ & 0.0008 & 0.00054 & 0.00078 \\
& $55-59$ & 0.0002 & 0.00008 & 0.0001
\end{tabular}

2011. ${ }^{[16]}$ This suggests that more adolescent females are utilising abortion services and, since they are surviving to report on it, they are utilising safe services.

Maternal mortality in SA is decreasing over time. This is a unique feature in SSA, with other countries in the region, such as Nigeria, Ethiopia and the Democratic Republic of the Congo, experiencing increasing maternal mortality rates. ${ }^{[19]}$ Healthcare facilities in SA have undergone major reform over the last 20 years, increasing access and affordability for the general population. ${ }^{[20]}$ In the area of obstetric care, the level of antenatal care visits in SA is high, with $97 \%$ of the 208 adult females interviewed in a Johannesburg study reporting that they sought care early in their pregnancy. ${ }^{[21]}$ And while access to care could be contributing to reduced maternal mortality, SA's declining fertility rate offers another possible explanation. The national total fertility rate has declined from 3.2 to 2.6 births between 1996 and 2012. ${ }^{[22,23]}$ However, the declining trend in SA does not signify that the country is on par with more developed countries. The maternal mortality rate in the USA is 29 deaths per 100000 live births and in Austria it is as low as 4 deaths per 100000 live births. ${ }^{[2]}$ This comparison proves that SA still has a way to go to further reduce maternal mortality. Despite the overall improvements in healthcare for pregnant adolescents in SA, incomplete medical histories and discrimination from hospital staff are also known to prevent them from seeking the necessary care. ${ }^{[25,26]}$

A systematic review of causes of maternal mortality found haemorrhage to be the leading cause of death in Africa. ${ }^{[27]}$ In SA though, the current study and another found gestational hypertension to be the leading cause of adolescent and adult maternal death. ${ }^{[28]}$ Risk factors associated with gestational hypertension are poor diet leading to gestational weight gain, pre-existing hypertension and diabetes. ${ }^{[29,30]}$ Specific to this are occurrences of pre-eclampsia among pregnant adolescents. Pre-eclampsia in adolescents is particularly dangerous, with a global prevalence of $\sim 1.9 \%$ and rates in Africa as high as $5.2 \% \cdot{ }^{[31]}$ Adolescents are at higher risk of pre-eclampsia than older females (20 - 29 years), and the known consequences include low birth weight and preterm delivery. ${ }^{[1,32]}$ Therefore, among pregnant females, preexisting conditions need to be frequently monitored as potential mortality hazards. This is offered at prenatal care visits. Yet, one study found that only $41 \%$ of pregnant adolescents access this service in SA. ${ }^{[33]}$

A second concerning cause of death affecting pregnant adolescents is abortion. 
The ICD-10 code for an abortion is inclusive of miscarriages, spontaneous abortion as well as termination of pregnancy. ${ }^{[34]}$ While termination of pregnancy (abortion) services have been legal in SA for almost 20 years, there remains a restriction on the accessibility of safe abortions for adolescents. In Africa it was found that 29 abortions per 1000 women were done in 2003 and $97 \%$ of these were unsafe. ${ }^{[35]}$ In SA, race, class, stigma and geographical location are known boundaries to women wishing to seek these services safely. As a result, they resort to quick, unsafe abortions that are not always done by trained service providers and are associated with an increased risk of complications. ${ }^{[36,37]}$ Globally, the proportion of maternal deaths due to unsafe abortions is $13 \%$; the proportion is higher in SSA at $14 \% .{ }^{[38]}$ In an effort to address the failings of abortion services in the country, an amendment was made to the SA Termination of Pregnancy Act. ${ }^{[39]}$ A result of this amendment was an increase in the number of facilities offering abortion services, from $31.5 \%$ in 2000 to almost $62 \%$ in $2003 .{ }^{[40]}$ Since then, definitive and official reports on the number of safe abortions conducted in the country have been moot. ${ }^{[39]}$ It is suggested that due to the cultural and religious significance surrounding pregnancy and termination thereof, conscientious objection by healthcare providers and admission staff at facilities has contributed to irregularity in the access and reporting of services. ${ }^{[41]}$

\section{Study strengths}

Firstly, the pooled data of 7 years of DNFs increased the size of the adolescent maternal deaths sample. This enabled a more robust quantitative and inferential analysis. The combined sample is also larger than any previous hospital-based records used in maternal mortality studies in SA. Secondly, the use of life tables makes it possible to estimate the contribution of specific causes of death to probability of dying by age. By using associated single decrement life tables, this study was able to identify the gains in survival if maternal causes of death were eliminated from the mortality experience of adolescent females. This has important policy and programme implications because it provides empirical proof of the need to reduce direct and indirect causes of maternal mortality. Lastly, this study addressed an issue that has been overlooked in adolescent health research in SA. Adolescent maternal mortality is occurring in the country, and this study has contributed to knowledge through identifying the levels and causes contributing to this occurrence which compromises the future social and economic development of the country.

\section{Study limitations}

Outside of hospital data, which is scarce, maternal mortality statistics are difficult to ascertain. DNFs have been used in this study to obtain national estimates of maternal mortality. However, this data source is limited in its capturing of pregnancy status at the time of death, with missing cases reported to be as high as $32.44 \%$. For this reason, the data have been pooled into a single dataset to increase the sample size and provide better estimates. In addition, the data do not account for deaths to females in the postnatal period (up to 42 days after giving birth); therefore the strict definition of maternal mortality, which includes this time period, could not be adhered to in this study. Finally, the data were subject to misclassification of causes of death, which is done at the level of medical practitioner, who completes the forms. In these cases women who died of tuberculosis, for example, could have been classified as 'maternal sepsis' owing to the similarity of symptoms of the diseases and the presence of the pregnancy. ${ }^{[42]}$

\section{Implications of the study}

There is a difference in the levels and causes of adolescent and adult maternal mortality in SA. This result is important because it means that the same approach to maternal care that is used for adults should not be used for adolescents. Adolescence is a pivotal developmental stage, whereby adolescents are gaining independence for the first time but are still reliant on care and tutelage. This makes the phase of adolescence markedly different from child- and adulthood. For this reason, age-sensitive approaches should be developed and monitored to address the specific needs of pregnant females at different ages in the country. Further, although rates are not as high for adolescents as for adult women, pregnant adolescents are still dying. In order to combat this, further research into the health-seeking challenges of pregnant adolescents should be conducted. And as not all pregnancies are wanted, resulting in stressful and harmful circumstances, research into the termination of pregnancy practices of adolescents would further assist in the reduction of hypertension and abortion as contributors to adolescent maternal mortality. Should gestational hypertension, abortion, violence and other direct and indirect causes of maternal mortality be removed from the mortality experience of pregnant adolescents, the probability of pregnant adolescents dying practically disappears. As the direct causes are all obstetric and pregnancy related, improved healthcare facilities, including the early and efficient detection of obstetric complications, will improve the survival of adolescents. Continued improvements to healthcare facilities in SA, paying special attention to youth obstetric care, particularly in the area of destigmatising adolescent pregnancy, and subsidising costs should remain a priority. Ensuring that hospitals are appropriately stocked and staffed with specialised care for pregnant adolescents and adults will reduce the number of maternal fatalities. Indirect causes of death can also be reduced through improving healthcare, but injury and non-communicable disease mortality can even more easily be reduced through behavioural change. The promotion of safe and healthy lifestyle choices through community engagement and education, especially among adolescents, could prevent unwanted pregnancies as well as any complications that may arise from these. In addition, healthy lifestyle choices encourage positive healthy behaviour, such as prenatal care visits. The health and development of pregnant adolescents are hindered by the risk of mortality associated with pregnancy. In recognising that these deaths are avoidable and unnecessary, appropriate measures can be designed and implemented to ensure the survival of adolescent females.

\section{References}

1. Jain V, Brown CS, Johnson O. Sierra Leone: The forgotten mortality. Glob Health Action 2015;8. DOI:10.3402/gha.v8.26757

2. National Committee for Confidential Enquiry into Maternal Deaths. Saving Mothers 2011 - 2013: Sixth report on confidential enquiries into maternal deaths in South Africa: Fact sheet. 2015. Pretoria: National Department of Health, 2015.

3. Buga EC, Nethathe GD, Mathivha LR. Obstetric critical care services in South Africa: Opinion. S Afr J Obstet Gynaecol 2015;21(1):4-5. DOI:10.7196/sajog.954

4. Statistics SA. Millennium Development Goals, Country Report 2013. Pretoria: Statistics SA, 2013.

5. Timæus IM, Moultrie TA. Teenage childbearing and educational attainment in South Africa. Stud Fam Plann 2015;46(2):143-160. DOI:10.1111/j.17284465.2015.00021.x

6. Willan S. A Review of Teenage Pregnancy in South Africa. Cape Town: Partners in Sexual Health, 2013.

7. Macleod CI. 'Adolescence', Pregnancy and Abortion: Constructing a Threat of Degeneration. Grahamstown, South Africa: Routledge, 2010.

8. Grant MJ, Hallman KK. Pregnancy-related school dropout and prior school performance in KwaZulu-Natal, South Africa. Stud Fam Plann 2008;39(4):369382. DOI:10.1111/j.1728-4465.2008.00181.x

9. Ross A, ed. 2015 National Conference on Health Communication, Marketing, and Media (August 11 - 13). In: Your Sexual Health Matters: A Media Campaign Targeting Adolescents. Atlanta, USA: Centers for Disease Control, 2015.

10. Zahr CA, Wardlaw TM. Maternal mortality in 2000: Estimates developed by WHO, UNICEF and UNFPA. Geneva: World Health Organization, 2004.

11. De Wet N, Odimegwu C. Determinants of adolescent mortality in South Africa, 2001 - 2007. J Behav Health 2013;2(3):243-251. DOI:10.5455/jbh. 20130726044607

12. Statistics SA. Recorded Live Births 2012. Pretoria: Statistics SA, 2013.

13. Granja ACL, Machungo F, Gomes A, Bergström S. Adolescent maternal mortality in Mozambique. J Adolesc Health 2001;28(4):303-306. DOI:10.1016/ s1054-139x(00)00205-6 
14. Bhutta ZA, Chopra M, Axelson H, et al. Countdown to 2015 decade report (2000 - 2010): Taking stock of maternal, newborn, and child survival. Lancet 2010;375(9730):2032-2044. DOI:10.1016/s0140-6736(10)60678-2

15. Graczyk K. Adolescent Maternal Mortality: An Overlooked Crisis. Washington, DC: Advocates For Youth, 2007.

16. Jonas K, Crutzen R, van den Borne B, Sewpaul R, Reddy P. Teenage pregnancy rates and associations with other health risk behaviours: A three-wave crosssectional study among South African school-going adolescents. Reprod Health 2016;13(1):1. DOI:10.1186/s12978-016-0170-8

17. Statistics SA. General household survey 2014. In: Africa SS, ed. South Africa General Household Survey 2014. Pretoria: Nesstar, 2015.

18. Althabe F, Moore JL, Gibbons L, et al. Adverse maternal and perinatal outcomes in adolescent pregnancies: The Global Network's Maternal Newborn Health Registry study. Reprod Health 2015;12(Suppl 2):S8. DOI:10.1186/1742-4755-12-s2-s8

19. Hogan MC, Foreman KJ, Naghavi M, et al. Maternal mortality for 181 countries, 1980 - 2008: A systematic analysis of progress towards Millennium Development Goal 5. Lancet 2010;375(9726):1609-1623. DOI:10.1016/s01406736(10)60518-1

20. Cooper D, Morroni C, Orner P, et al. Ten years of democracy in South Africa: Documenting transformation in reproductive health policy and status. Reprod Health Matters 2004;12(24):70-85. DOI:10.1016/s0968-8080(04)24143-x

21. Solarin I, Black V. 'They told me to come back': Women's antenatal care booking experience in inner-city Johannesburg. Matern Child Health J 2013;17(2):359367. DOI:10.1007/s10995-012-1019-6

22. Statistics SA. Mid-Year Population Estimates 2015. Pretoria: Statistics SA, 2015.

23. Moultrie TA, Timæus IM. The South African fertility decline: Evidence from two censuses and a demographic and health survey. Popul Stud 2003;57(3):265283. DOI:10.1080/0032472032000137808

24. World Bank. Maternal Mortality Ratios (modeled estimate, per 100,000 live births). Washington, DC: World Bank Group, 2015. http://data.worldbank. org/indicator/SH.STA.MMRT?order=wbapi_data_value_2013+wbapi_data_ value+wbapi_data_value-last\&sort=asc (accessed 21 June 2015 ).

25. Wiemann $C M$, Rickert VI, Berenson $A B$, Volk RJ. Are pregnant adolescents stigmatized by pregnancy? J Adolesc Health 2005;36(4):352,e1-e7. DOI:10.1016/ j.jadohealth.2004.06.006

26. Jewkes R, Abrahams N, Mvo Z. Why do nurses abuse patients? Reflections from South African obstetric services. Soc Sci Med 1998;47(11):1781-1795. DOI:10.1016/s0277-9536(98)00240-8

27. Khan KS, Wojdyla D, Say L, Gülmezoglu AM, van Look PF. WHO analysis of causes of maternal death: A systematic review. Lancet 2006;367(9516):10661074. DOI:10.1016/s0140-6736(06)68397-9
28. Soma-PillayP,PattinsonR,Langa-MlamboL,NkosiB,MacdonaldA.Maternalnear miss and maternal death in the Pretoria Academic Complex, South Africa: A population-based study. S Afr Med J 2015;105(7):578-583. DOI:10.7196/ saminew.8038

29. Swank M, Caughey A, Farinelli C, et al. The impact of change in pregnancy body mass index on the development of gestational hypertensive disorders. J Perinatol 2014;34(3):181-185. DOI:10.1038/jp.2013.168

30. Wang I-K, Muo C-H, Chang Y-C. Association between hypertensive disorders during pregnancy and end-stage renal disease: A population-based study. Can Med Assoc J 2013;185(3):207-213. DOI:10.1503/cmaj.120230

31. Ganchimeg T, Ota E, Morisaki N, et al. Pregnancy and childbirth outcomes among adolescent mothers: A World Health Organization multicountry study. Br J Obstet Gynaecol 2014;121(s1):40-48. DOI:10.1111/1471-0528.12630

32. Eure CR, Lindsay MK, Graves WL. Risk of adverse pregnancy outcomes in young adolescent parturients in an inner-city hospital. Am J Obstet Gynecol 2002;186(5):918-920.

33. McCray TM. An issue of culture: The effects of daily activities on prenatal care utilization patterns in rural South Africa. Soc Sci Med 2004;59(9):18431855. DOI:10.1067/mob.2002.123986

34. American Academy of Professional Coders. ICPD -10 Overview. http://www. aapccom/ICD-10/icd-10aspx (accessed 28 April 2011).

35. Sedgh G, Henshaw S, Singh S, Åmman E, Shah IH. Induced abortion: Estimated rates and trends worldwide. Lancet 2007;370(9595):1338-1345. DOI:10.1016/s0140-6736(07)61575-x

36. Varkey SJ. Abortion services in South Africa: Available yet not accessible to all. Int Fam Plan Perspect 2000;26(2):87-88. DOI:10.2307/2648273

37. Jacobs R, Hornsby N. Why aren't women getting safe abortions? S Afr Med J 2014;104(12):857-858. DOI:10.7196/samj.9133

38. Åhman E, Shah IH. New estimates and trends regarding unsafe abortion mortality. Int J Gynecol Obstet 2011;115(2):121-126. DOI:10.1016/j. ijgo.2011.05.027

39. Hodes R. The culture of illegal abortion in South Africa. J South Afr Stud 2016;42(1):79-93. DOI:10.1080/03057070.2016.1133086

40. Health Systems Trust. ToP Facilities Functioning. Cape Town: Health Systems Trust, 2012. http://www.hst.org.za/news/half-sa-pregnancies-end-abortion (12 August 2015).

41. Harries J, Stinson K, Orner P. Health care providers' attitudes towards termination of pregnancy: A qualitative study in South Africa. BMC Public Health 2009;9(1):1. DOI:10.1186/1471-2458-9-296

42. Acosta CD, Knight M. Sepsis and maternal mortality. Curr Opin Obstet Gynecol 2013;25(2):109-116. DOI:10.1097/gco.0b013e32835e0e82 